\title{
Approaches to Bayesian network structure elicitation
}

\author{
K. Zwirglmaier \& D. Straub \\ Engineering Risk Analysis Group, Technische Universität München, Germany
}

\begin{abstract}
Bayesian networks $(\mathrm{BN})$ are increasingly popular as a modeling tool for engineering risk and reliability analysis. BNs consist of two parts: the qualitative dependence structure represented by a directed acyclic graph (DAG), and the quantification of local dependences through conditional probability distributions. We review the qualitative modeling, the elicitation of the $\mathrm{BN}$ structure, and classify existing approaches into four categories: (1) the transformation from existing probabilistic models such as fault trees, event trees; (2) the derivation from existing physical or empirical models; (3) structure elicitation based on domain expert knowledge; (4) structure elicitation based on data. This paper gives general descriptions of these structure elicitation approaches and discusses how they are applied in the field of engineering risk analysis. The paper ends with a discussion of the challenges in BN structure elicitation.
\end{abstract}

\section{INTRODUCTION}

The performance of most engineering systems is associated with uncertainties and risk. In many domains, these are addressed by a probabilistic risk assessment. In such an assessment, domain experts in the specific disciplines and probability experts have to work closely together. A well-suited modeling framework for this purpose is the $\mathrm{BN}$, which on the one hand is flexible enough to represent a wide variety of probabilistic dependencies, and on the other hand allows nonprobability experts to understand the basic dependence structure encoded in the models through the BNs graphical form.

BNs have been successfully applied as a tool for reasoning under uncertainty in various fields including machine learning (Bishop, 2006), medicine (Lucas, 2001), law (Fenton and Neil, 2000), environmental modeling (Aguilera et al., 2011) environmental and resource management (Barton et al., 2012), reliability (Langseth and Portinale, 2007, Weber et al., 2012).

BNs consist of a qualitative part, the dependence structure represented by a directed acyclic graph (DAG), and a quantitative part, the local conditional probability distributions (CPDs). For discrete BNs the latter are given in the form of conditional probability tables (CPTs). The derivation of the general dependence structure requires a profound understanding of the problem as well as an understanding of the modeling tool. Once the general structure is developed, the probability expert knows what CPDs need to be elicited through data, expert estimates or a combination of both.

In the field of engineering risk and reliability analysis, the structure elicitation process by itself has received little attention. Langseth and Portinale (2007) explain application of BNs to reliability including quantitative and qualitative model building and inference. They give some suggestions on BN elicitation based on domain experts and explain how BNs can replace fault trees (FTs). (Fenton and Neil, 2012) propose idioms intended for supporting $\mathrm{BN}$ experts in model building. (Kjaerulff and Madsen, 2013) distinguish between different types of variables and describe generic high-level dependence structures between these types of variables. Finally (Conrady and Jouffe, 2015) give some insight into how BN structures can be elicited.

Based on an extensive literature review, we distinguish four approaches for $\mathrm{BN}$ structure elicitation, namely: (1) transformation from existing probabilistic models such as fault trees, event trees; (2) derivation from existing physical or empirical models; (3) structure elicitation based on data; (4) structure elicitation based on domain expert knowledge. Each of these four approaches is presented in the following sections together with a small application from the field of engineering risk 
analysis. In the final discussion, typical challenges associated with the approaches are discussed.

\section{BN STRUCTURES BASED ON OTHER PROBABILISTIC MODELS}

In many industries, probabilistic models like fault trees (FTs) or event trees (ETs) have become popular due to their simplicity and the possibility to standardize their elicitation. Since BNs are more flexible, they are increasingly replacing or enhancing these models. In such a process, as a first step, a BN is derived from existing FTs, ETs or other probabilistic models.

The mapping of FTs and ETs to BNs is considered in the following. This process is rather intuitive and straightforward.

\subsection{Fault trees (FTs)}

FTs are typically used to break down system failure events into their constituent basic events. Boolean operators (gates) are used to link one level of events to the next. The most basic operators are the AND and the OR gate - other gate types are available e.g. (Vesely et al., 1981, Villemeur, 1992). Both AND and $\mathrm{OR}$ gates can be represented in a $\mathrm{BN}$ through a converging structure (Fig. 1), the CPTs of the child node are as shown in Fig. 1d and e. Transformation of FTs to BNs has been described by many authors, including (Bobbio et al., 2001, Mahadevan et al., 2001).

\subsection{Event trees (ETS)}

While FTs model the occurrence of a top (failure) event starting from several initiating events, ETs model the paths from the top event to the possible final states (represented by the random variable $Y$ in Fig. 2). Often FTs and ETs are used in combination in so-called bow-tie models, where the top event from the FT represents the initiating event of the ET (Andersen et al., 2004). Transformation of ETs to BNs has been described e.g. in (Bearfield and Marsh, 2005). An ET is shown in Fig. 2a. The ordering of events from left to right typically follows the causal direction, which is evident in the corresponding BN shown in Fig. 2b. Whether a link between any two nodes is actually present can be inferred from the ET, which includes the complete joint probability distribution. a) AND gate in a FT

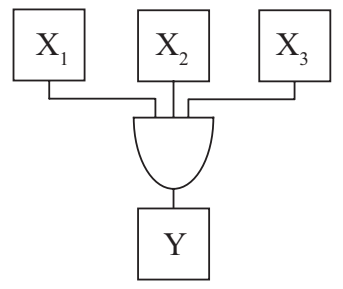

b) OR gate in FT

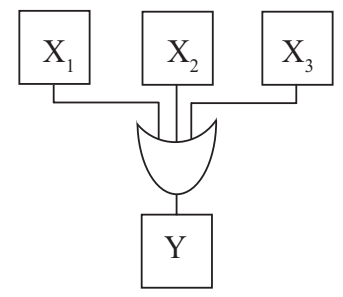

c) Representation of the FT gates in a $\mathrm{BN}$ structure

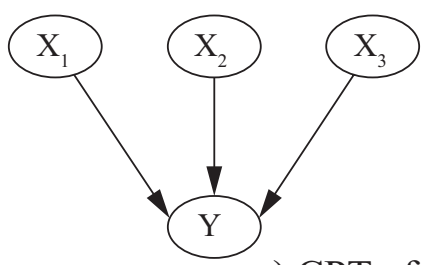

d) CPT of $\mathrm{Y}$ for AND gate

e) CPT of $\mathrm{Y}$ for OR gate

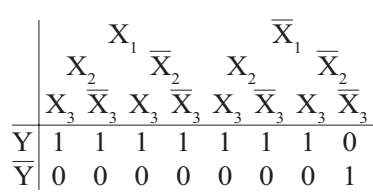

Figure 1. Transformation of an AND resp. OR gate from a FT to a $\mathrm{BN}$.

\subsection{Application example}

A simple ET for determining the probability of fatalities as a consequence of a ship accident is shown in Fig. 3, adapted from IMO (2010). The random variables in the ET, namely operational state, damage extend and fatalities are represented through nodes in the BN. Potentially all nodes in the $\mathrm{BN}$ can be connected. Since the order of variables in the ET from left to right is causal, the directions of the potential links are predetermined. Whether two nodes are actually connected can be derived from the probabilities in the ET. Based on the probability distributions in the ET there exists a link from Operational state to Damage extent as well as from Damage extent to Fatalities. No link is present between Operational state and Fatalities, because these are independent conditional on knowing the state of Damage extent, i.e. $p$ (Fatalities $\mid$ Op. state, Damage extent $)=$ $p$ (Fatalities| Damage extent). 


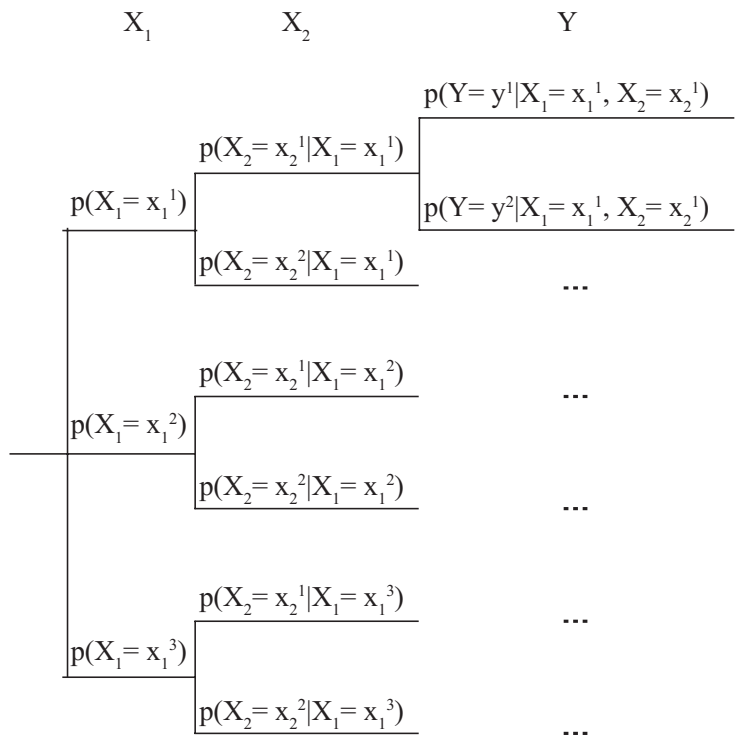

a) Event tree with two intermediate random variables $\mathbf{X}=\left\{X_{1}, X_{2}\right\}$ and a final random variable $\mathrm{Y}$

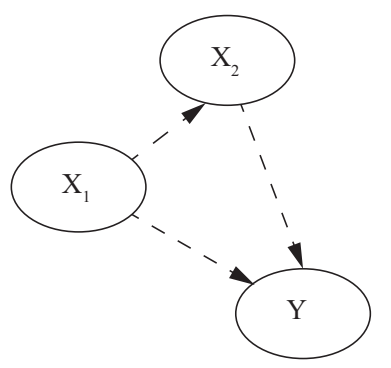

b) $\mathrm{BN}$ representation of the event tree

Figure 2. Transformation of an ET to a BN.

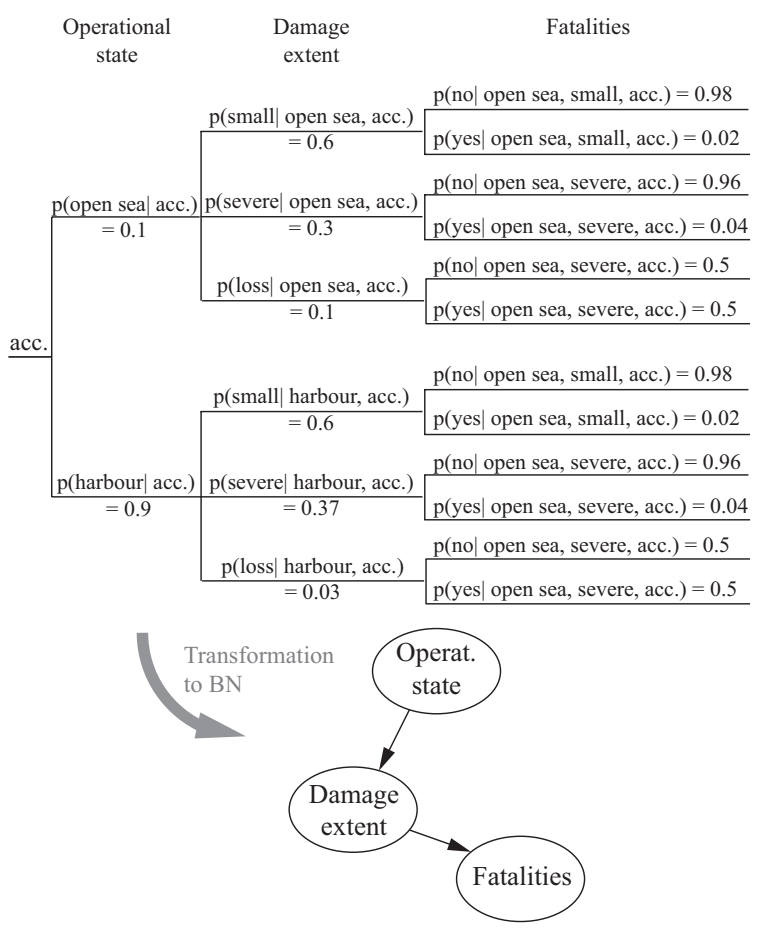

Figure 3. Transformation of an ET for determining the consequences of a ship accident to a BN.

\section{BN STRUCTURES BASED ON PHYSICAL AND EMPIRICAL MODELS}

Often physical or empirical models exist of the processes leading to failure and risk. In combination with a stochastic model of its input parameters, such models can be used in a probabilistic risk analysis. To enable a fast updating of the model with new information or to extend the model, it may be desirable to represent the model in a BN (Straub and Der Kiureghian, 2010).

\subsection{Representation in a $B N$}

A model $y=g(\mathbf{x})$ with independent random variables $\mathbf{X}=\left[\mathrm{X}_{1}, \ldots, X_{n}\right]$ can be represented in a $\mathrm{BN}$ as shown in Fig. 4. Dependence between the random variables in $\mathbf{X}$ can be modeled through directed links between pairs of them or through common parents. The model $g$ can be analytical or numerical.

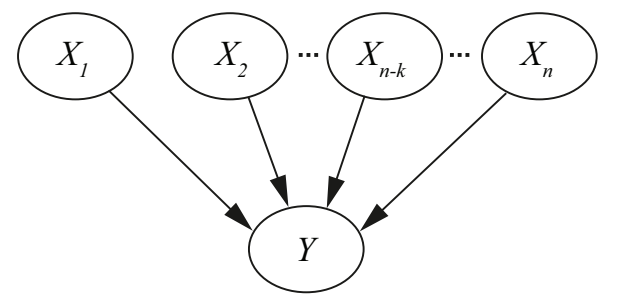

Figure 4. BN representation of a physical/empirical model.

For models with many basic random variables $\mathbf{X}$, it can be computationally unfeasible to include all random variables $\mathbf{X}$ explicitly in the $\mathrm{BN}$. In such cases, some of the random variables can be considered implicitly in the BN (Zwirglmaier and Straub, 2015b). This corresponds to integrating $g(\mathbf{X})$ over the random variables $\mathbf{X}^{\text {imp }}$ (with $\mathbf{X}^{\text {imp }} \subset \mathbf{X}$ ) that are to be modeled implicitly.

\subsection{Application example}

Following (Drees and Holzapfel, 2012) the landing distance required by a landing aircraft can be determined from variables such as approach speed deviation, head wind, landing weight and multiple other parameters through a numerical model $g$. In flight these parameters are not known with certainty and are thus modeled by random variables, $\mathbf{X}$.

The model is transformed to a $\mathrm{BN}$ following (Zwirglmaier and Straub, 2015b). The resulting BN is shown in Fig. 5. Only approach speed deviation, head wind, landing weight are modeled explicitly and all other random variables are treated as implicit uncertainties, since they are either not important or not observable at the required point of time. The model structure can be extended e.g. based on 
domain expert knowledge. This is indicated through the grey nodes airport and aircraft-type in Fig. 5. These nodes causally influence the basic random variables of the physical model.

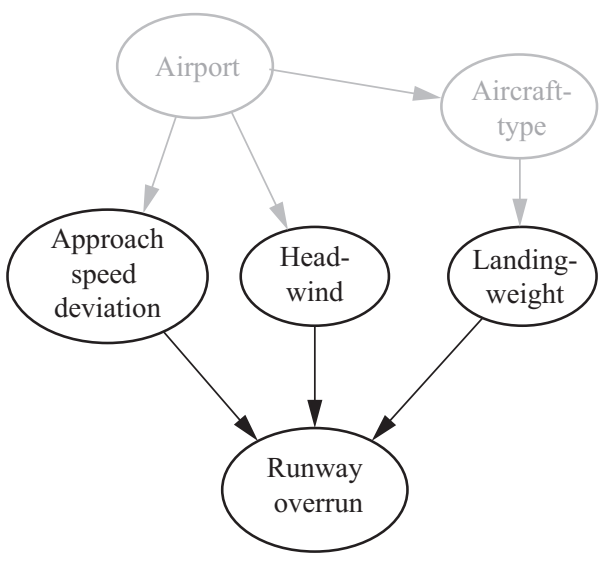

Figure 5. BN derived from a physical model RWO = $g$ (Approach speed dev. , Head wind, Landing weight, ... ) (Zwirglmaier and Straub, 2015a).

\section{BN STRUCTURES BASED ON DOMAIN EXPERT KNOWLEDGE}

For problems, for which neither another probabilistic models nor a physical/empirical model exist, or the existing models do not meet the modeler's requirements, a $\mathrm{BN}$ structure can be elicited directly from domain expert knowledge.

\subsection{Modeling approach}

The procedure for eliciting BNs from domain expert can be structured into the following three steps:

1. Problem clarification and identification of relevant parameters

2. Modeling of relevant dependencies

3. Handling unquantifiable variables

\subsubsection{Problem clarification and identification of relevant variables}

Before building the model, the relevant variables have to be determined. These are the variables of interest (the problem variables in the notation of Kjaerulff and Madsen (2013)), the information variables (the observable parameters) and the mediating variables, which are not observable but help in representing the dependencies between information variables and the problem variables. This identification of the relevant variables typically cannot be treated independently from the dependence structure. Each of the relevant variables should be clearly defined including its type, support and temporal and spatial reference.

Methods such as organized interviews (Hanea and Ale, 2009), failure mode and effect analysis
(FMEA) (Suddle, 2009, Weber and Jouffe, 2006, De Carlo et al., 2013), methods from system engineering like structured analysis and design technique (SADT) (Trucco et al., 2008, Weber and Jouffe, 2006, De Carlo et al., 2013) can be used to support the identification of relevant variables and their dependencies.

\subsubsection{Modeling of relevant dependencies}

The dependence structure among the relevant parameters has to be introduced through directed links. Though not required by $\mathrm{BN}$ theory, the dependencies ideally represent causality, since this typically leads to more efficient and better traceable models. A simple example for causality is a result from a structural monitoring that indicates a poor condition of the structure. There are two possible ways for orienting the link between the two variables.

I) From monitoring result to condition of the structure: This is referred to as the diagnostic direction i.e. by looking at the monitoring result one concludes that the condition of the structure is poor.

II) From condition of the structure to monitoring result: This is referred to as the causal direction i.e. the condition of the structure determines the monitoring result.

Option I is often chosen intuitively, but option II is preferable for modelling and computation purposes. Often the causal direction of the link may be assessed from the temporal order; following (Pearl, 2009), "a later event can never be the cause of an earlier event". To facilitate causal BN structure elicitation (Neil et al., 2000, Fenton and Neil, 2012) propose the use of (four) idioms. Kjaerulff and Madsen (2013) propose general dependence structures that represent how the types of variables (i.e. problem, information and mediating random variables) are typically connected.

\subsubsection{Unquantifiable variables}

The qualitative BN model can contain unquantifiable variables. These should be dealt with separately. Unquantifiable variables are those for which no reasonable probability estimates can be obtained from experts or data. Note that having no data on a particular node does not necessarily imply that it cannot be quantified. The EM algorithm may under certain circumstances allow estimating the CPDs of nodes with no data (Lauritzen, 1995).

(Hanea et al., 2006, Hanea and Ale, 2009) propose to replace unquantifiable variables by proxy variables that are quantifiable. In cases where it is 
expected that data or expert knowledge for quantifying the unquantifiable variable will become available in the near future it may be sufficient to use dummy parameters for the preliminary quantification. However if this information is not expected to be available, these variables should be eliminated from the network to make the BN applicable in practice. In (Zwirglmaier et al., 2015) we use node removal algorithms for this purpose, as proposed in (Shachter, 1988). Straub and Der Kiureghian (2010) applied these algorithms to remove continuous variables in their enhanced $\mathrm{BN}$ framework.

Node reduction algorithm (Shachter, 1988):

- Barren nodes, i.e. the ones which have no children and which have not received evidence can be removed from the network.

- To make nodes barren, arcs can be reversed. In order to revers an arc between two nodes $X_{i}$ and $X_{j}$, both nodes must inherit each others parents, without making the directed graph cyclic.

The presented node reduction algorithm ensures that the (in-)dependence properties between the remaining random variables are not altered. It should be noted that the reduced structure depends on the order of node removals. Furthermore node reduction algorithms may lead to nodes having more parents than in the original (qualitative) network.

\subsection{Application example}

The misperception of critical data in the control room of a nuclear power plant can have severe consequences. Attention degradation is a possible cause of such misperception, as described in the literature on cognitive psychology (Whaley et al., 2012, Xing et al., 2013).

Attention degradation may come from crew members having biased expectations or prioritizing falsely. Both issues can be influenced through missing or faulty training. Prioritization is furthermore influenced by the perception of urgency of the crew. Finally, attention degradation can be fostered by high workloads.

The resulting relevant parameters and the dependence structure are summarized in the BN of Fig. 6 (adapted from Zwirglmaier et al. (2015)). Some of the parameters are not quantifiable with feasible effort. These nodes are marked in white in Fig. 6. Shachter's node reduction algorithm is applied to remove them from the BN. The resulting BN structure is shown in Fig. 6.

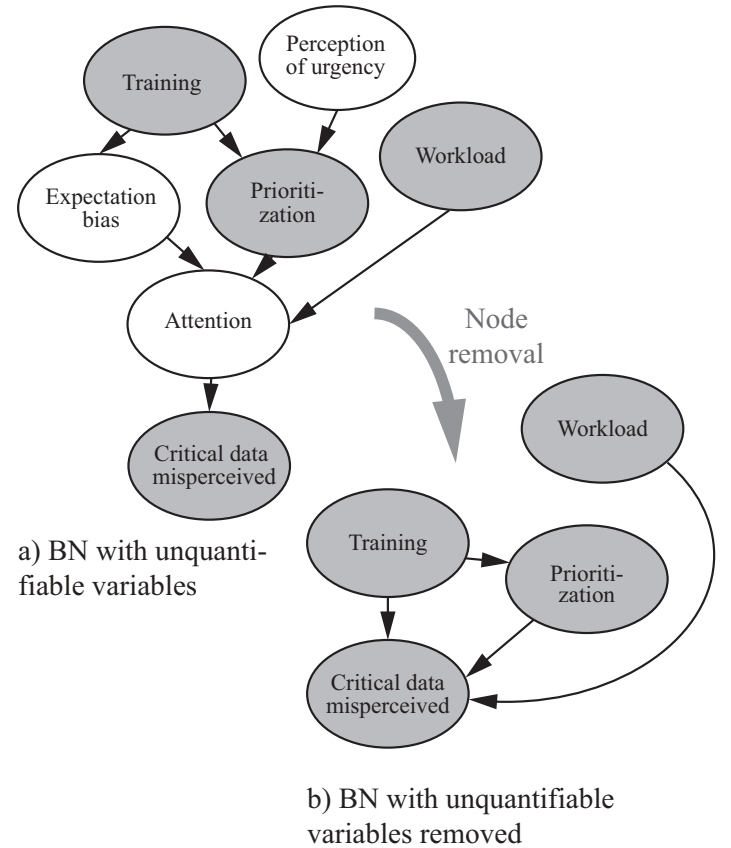

Figure 6. BN structure for critical data misperceived (Zwirglmaier et al., 2015). The unquantifiable (white) nodes are removed from the network.

\section{DATA BASED STRUCTURE LEARNING}

If sufficient data on the parameters of interest are available, the BN structure can be directly learned from the data.

\subsection{Modeling approach}

There are two approaches for structure learning based on data. Constraint-based approaches make use of statistical independence tests to derive a set of independence statements from a dataset. From these independence statements, a BN structure is constructed. Examples of constrained-based algorithms are the SGS (Spirtes et al., 1989), the PC (Spirtes et al., 2001) and the NPC algorithm (Steck, 2001).

In the score-based approach, a number of candidate structures are derived and tested using a scoring function. Since the number of possible BN structures grows faster than exponentially with the number of nodes (Robinson, 1977), an exhaustive search is infeasible and efficient search heuristics have to be applied. Examples of scoring functions are the Akaike information criterion (AIC) by Akaike (1974), the closely related Bayesian information criterion (BIC) by Schwarz (1978), the Bayesian Dirichlet (BD) scores (Heckerman et al., 1995) or the maximum a posteriori (MAP BN) criterion (Riggelsen, 2008). Examples of search approaches are greedy search algorithms such as the repeated covered arc reversal algorithm (Castelo and Kocka, 2003), the search over equivalent BN classes 
(Chickering, 2002) or a search over node orderings (Teyssier and Koller, 2012).

One distinguishes unsupervised and supervised structure learning. Unsupervised approaches aim at finding the BN model that represents the learningdataset best. Supervised approaches aim at finding a model that is best suited for predicting one or more pre-selected target variables. BNs for which additionally only the state of the target variable with the highest posterior-probability is of interest are referred to as classifiers (Friedman et al., 1997). For classification tasks, structures are not learned (or only partly learned), since predefined structures such as Naïve Bayesian classifiers (NBCs) or Tree Augmented Naïve BNs (TANs) have shown to be efficient (Zhang, 2004). However, classification is of limited interest in risk analysis, where typically the entire posterior distribution is of relevance.

\subsection{Application example}

The BN in Fig. 7a represents a model for the daily avalanche probability in a specific area. Indicators used for the prediction are the season (spring, summer, fall and winter), the vegetation (dense trees, no dense trees), the slope of the terrain $\left(<30^{\circ}, 30^{\circ}-45^{\circ},>45^{\circ}\right)$. The avalanche probability in summer as well as for slopes $<30^{\circ}$ or $>45^{\circ}$ is assumed to be 0 . The maximal avalanche probability of 0.15 corresponds to terrains between $30^{\circ}-45^{\circ}$ with no dense tree vegetation in winter. The avalanche probabilities in fall (winter) are two (five) times higher than in spring and dense trees decrease avalanche probabilities by a factor of three. The probability distributions of the nodes slope and season are uniform; the one of vegetation is defined conditional on slope, the probability of having dense tree vegetation is $[0.3,0.5,0]$ for slopes of $\left[<30^{\circ}, 30^{\circ}-45^{\circ},>45^{\circ}\right]$.

From the BN in Fig. 7a, 500 samples are generated. These are used in a score based structure learning approach, implemented as a greedy search strategy with the BIC scoring function. From these samples the influence of season and slope on avalanche probability is identified correctly, but not the influence of vegetation. The dependence between slope and vegetation is also identified from the data, but in the non-causal direction. However, the implied (in-)dependence criteria are correct, and it is therefore not possible to identify the direction of this link based on data alone. Also, the direction of this link does not affect predictions.
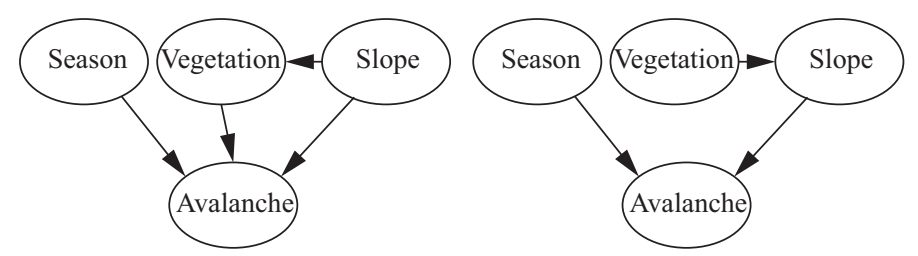

Figure 7. (a) Original BN for predicting avalanche probability. (b) BN learnt with 500 samples using a score based structure learning approach.

\section{DISCUSSION}

Approaches to $\mathrm{BN}$ structure elicitation have been categorized in four classes. Each of these approaches comes with own challenges, which are outlined briefly in this section.

The derivation of a $\mathrm{BN}$ structure from another probabilistic model is typically straightforward. For certain standardized settings, software exists for this task.

The derivation of an initial $\mathrm{BN}$ structure from a physical or empirical model is trivial. The main challenge is typically computational feasibility, in particular if the model has a larger number of uncertain input parameters $\mathbf{X}$. Strategies to address these issues include:

- Treating some of the random variables in $\mathbf{X}$ as implicit uncertainty in the $\mathrm{BN}$ (section 3.1).

- Applying more efficient BN structure representations e.g. (Heckerman and Breese, 1994, Bensi et al., 2013).

- Compression of CPTs to save memory space e.g. (Tien and Der Kiureghian, 2015).

- The use of sampling-based instead of exact inference algorithms.

For BNs based on domain expert knowledge, computational issues may arise e.g. if experts model a node with too many parents. This should be avoided in the model building process. If the parameters of a BN are to be elicited from experts or a small dataset, large CPTs are critical. To ensure that the assumed (in-)dependence assumptions are correctly represented by the $\mathrm{BN}$ graph, it is advisable to orient the links strictly according to their causal directions.

In BNs that are learnt from data the links do typically not follow the causal directions. On the one hand this makes reviewing the validity of the (in)dependence assumptions in the $\mathrm{BN}$ on the other hand extending of the $\mathrm{BN}$ challenging. 
Although the four structure elicitation approaches were presented individually, medium sized to large BNs are typically not developed solely through on one approach, but are based on a combination of them. Domain expert knowledge can be used to introduce additional parameters and dependencies to models that are based on physical/empirical models or on other probabilistic models. Separate model parts derived with different approaches can be combined to estimate the overall risk. The modularity of the $\mathrm{BN}$ facilitates such an integration. However, if not all model parts are causal BNs, such an integration requires great care to ensure that the $\mathrm{d}$-separation properties are accurately represented.

\section{CONCLUSION}

BNs are a probabilistic modeling framework that is flexible enough to represent various types of dependencies while also facilitating the communication with non-probability experts. In this paper, we categorize approaches for $\mathrm{BN}$ structure elicitation into four classes, which are reviewed. Applications from the field of engineering risk analysis are presented and the challenges typically encountered in such applications are briefly discussed.

\section{REFERENCES}

Aguilera, P. A., Fernández, A., Fernández, R., Rumí, R. \& Salmerón, A. 2011. Bayesian Networks In Environmental Modelling. Environmental Modelling \& Software, 26, 1376-1388.

Akaike, H. 1974. A New Look At The Statistical Model Identification. Automatic Control, Ieee Transactions On, 19, 716-723.

Andersen, H., Casal, J., Dandrieux, A., B, D., De Dianous, V., Duijm, N., Delvosalle, C., Fievez, C., Goossens, L., Gowland, R., Hale, A., Hourtolou, D., Mazzarotta, B., Pipart, A., Planas, E., Prats, F., Salvi, O. \& Tixier, J. 2004. Aramis User Guide European Comission Joint Research Centre Institute For The Protection And Security Of The Citizen.

Barton, D. N., Kuikka, S., Varis, O., Uusitalo, L., Henriksen, H. J., Borsuk, M., De La Hera, A., Farmani, R., Johnson, S. \& Linnell, J. D. 2012. Bayesian Networks In Environmental And Resource Management. Integrated Environmental Assessment And Management, 8, 418-429.

Bearfield, G. \& Marsh, W. 2005. Generalising Event Trees Using Bayesian Networks With A Case Study Of Train Derailment. Computer Safety, Reliability, And Security. Springer.
Bensi, M., Der Kiureghian, A. \& Straub, D. 2013. Efficient Bayesian Network Modelling Of Systems. Reliability Engineering \& System Safety, 112, 200-213.

Bishop, C. M. 2006. Pattern Recognition And Machine Learning, Springer.

Bobbio, A., Portinale, L., Minichino, M. \& Ciancamerla, E. 2001. Improving The Analysis Of Dependable Systems By Mapping Fault Trees Into Bayesian Networks. Reliability Engineering \& System Safety, 71, 249-260.

Castelo, R. \& Kocka, T. 2003. On Inclusion-Driven Learning Of Bayesian Networks. The Journal Of Machine Learning Research, 4, 527-574.

Chickering, D. M. 2002. Learning Equivalence Classes Of Bayesian-Network Structures. The Journal Of Machine Learning Research, 2, 445-498.

Conrady, S. \& Jouffe, L. 2015. Bayesian Networks And Bayesialab: A Practical Introduction For Researchers, Franklin, Tn, Bayesia Usa.

De Carlo, F., Borgia, O. \& Tucci, M. 2013. Imperfect Maintenance Modelling By Dynamic Object Oriented Bayesian Networks. International Journal Of Engineering And Technology, 5, 4282-4295.

Drees, L. \& Holzapfel, F. 2012. Determining And Quantifying Hazard Chains And Their Contribution To Incident Probabilities In Flight Operation. Aiaa Modeling And Simulation Technologies Conference. American Institute of Aeronautics And Astronautics.

Fenton, N. \& Neil, M. 2000. The Jury Observation Fallacy And The Use Of Bayesian Networks To Present Probabilistic Legal Arguments. Mathematics Today-Southend On Sea-, 36, 180-187.

Fenton, N. \& Neil, M. 2012. Risk Assessment And Decision Analysis With Bayesian Networks, Crc Press.

Friedman, N., Geiger, D. \& Goldszmidt, M. 1997. Bayesian Network Classifiers. Machine Learning, 29, 131-163.

Hanea, D. \& Ale, B. 2009. Risk Of Human Fatality In Building Fires: A Decision Tool Using Bayesian Networks. Fire Safety Journal, 44, 704-710.

Hanea, D., Ale, B. \& Cooke, R. The Methodology To Build The Network Used In A Bayesian Belief Net Approach (Psam-0068). Proceedings Of The Eighth International Conference On Probabilistic Safety Assessment \& Management (Psam), 2006. Asme Press.

Heckerman, D. \& Breese, J. S. 1994. A New Look At Causal Independence. Proceedings of The Tenth International Conference On Uncertainty In Artificial Intelligence. Seattle, Wa: Morgan Kaufmann Publishers Inc.

Heckerman, D., Geiger, D. \& Chickering, D. M. 1995. Learning Bayesian Networks: The 
Combination Of Knowledge And Statistical Data. Machine Learning, 20, 197-243.

Imo 2010. Iacs Fsa Study - Step 2 (Risk Analysis). General Cargo Ship Safety. International Maritime Organization - Maritime Safety Committee.

Kjaerulff, U. B. \& Madsen, A. L. 2013. Bayesian Networks And Influence Diagrams: A Guide To Construction And Analysis, Springer Publishing Company, Incorporated.

Langseth, H. \& Portinale, L. 2007. Bayesian Networks In Reliability. Reliability Engineering \& System Safety, 92, 92-108.

Lauritzen, S. L. 1995. The Em Algorithm For Graphical Association Models With Missing Data. Computational Statistics \& Data Analysis, 19, 191-201.

Lucas, P. 2001. Bayesian Networks In Medicine: A Model-Based Approach To Medical Decision Making, Na.

Mahadevan, S., Zhang, R. \& Smith, N. 2001. Bayesian Networks For System Reliability Reassessment. Structural Safety, 23, 231251.

Neil, M., Fenton, N. \& Nielson, L. 2000. Building Large-Scale Bayesian Networks. The Knowledge Engineering Review, 15, 257284.

Pearl, J. 2009. Causality, Cambridge University Press.

Riggelsen, C. 2008. Learning Bayesian Networks: A Map Criterion For Joint Selection Of Model Structure And Parameter. Icdm 2008. Pisa.

Robinson, R. W. 1977. Counting Unlabeled Acyclic Digraphs. Combinatorial Mathematics $V$. Springer.

Schwarz, G. 1978. Estimating The Dimension Of A Model. The Annals Of Statistics, 6, 461-464.

Shachter, R. D. 1988. Probabilistic Inference And Influence Diagrams. Oper. Res., 36, 589-604.

Spirtes, P., Glymour, C. N. \& Scheines, R. 1989. Causality From Probability.

Spirtes, P., Glymour, C. N. \& Scheines, R. 2001. Causation, Prediction, And Search, Cambridge. Mass. [U.A.], Mit Press.

Steck, H. 2001. Constraint Based Structural Learning In Bayesian Networks Using Finite Data Sets, Tu München, Phd Thesis.

Straub, D. \& Der Kiureghian, A. 2010. Bayesian Network Enhanced With Structural Reliability Methods: Methodology. Journal Of Engineering Mechanics, 136, 1248-1258.

Suddle, S. 2009. The Risk Management Of Third Parties During Construction In Multifunctional Urban Locations. Risk Analysis, 29, 1024-1040.

Teyssier, M. \& Koller, D. 2012. Ordering-Based Search: A Simple And Effective Algorithm For Learning Bayesian Networks. Arxiv Preprint Arxiv: 1207.1429.

Tien, I. \& Der Kiureghian, A. 2015. Compression And Inference Algorithms For Bayesian Network Modeling Of Infrastructure Systems.
Trucco, P., Cagno, E., Ruggeri, F. \& Grande, O. 2008. A Bayesian Belief Network Modelling Of Organisational Factors In Risk Analysis: A Case Study In Maritime Transportation. Reliability Engineering \& System Safety, 93, 845-856.

Vesely, W. E., Goldberg, F. F., Roberts, N. H. \& Haasl, D. F. 1981. Fault Tree Handbook. Dtic Document.

Villemeur, A. 1992. Reliability, Availability, Maintainability And Safety Assessment, Assessment, Hardware, Software And Human Factors, Wiley.

Weber, P. \& Jouffe, L. 2006. Complex System Reliability Modelling With Dynamic Object Oriented Bayesian Networks (Doobn). Reliability Engineering \& System Safety, 91, 149-162.

Weber, P., Medina-Oliva, G., Simon, C. \& Iung, B. 2012. Overview On Bayesian Networks Applications For Dependability, Risk Analysis And Maintenance Areas. Engineering Applications Of Artificial Intelligence.

Whaley, A., Xing, J., Boring, R. L., Hendrickson, S., Joe, J. \& Le Blanc, K. 2012. Building A Psychological Foundation For Human Reliability Analysis (Nureg-2114). Washington Dc: Us Nuclear Regulatory Commission.

Xing, J., Presley, M., Parry, G., Forester, J., Hendrickson, S. \& Dang, V. 2013. Nrc/Epri Draft Report For Peer Review: An Integrated Decision-Tree Human Event Analysis System (Idheas) Method For Npp Internal At-Power Operation. Nrc/Epri

Zhang, H. 2004. The Optimality Of Naive Bayes. $A a, 1$.

Zwirglmaier, K. \& Straub, D. 2015a. Discretization Of Structural Reliability Problems - An Application To Runway Overrun. Icasp 12. Vancouver, Canada.

Zwirglmaier, K. \& Straub, D. 2015b. A Discretization Procedure For Rare Events In Bayesian Networks. Submitted To Reliability Engineering And System Safety.

Zwirglmaier, K., Straub, D. \& Groth, K. 2015. Capturing Cognitive Causal Paths In Human Reliability Analysis With Bayesian Network Models. Submitted To Reliability Engineering And System Safety. 